\title{
UNA CASA DEL SIGLO XX Y UN FUERTE DEL SIGLO XVI. Arqueología histórica en Cobos
}

\author{
Jorge Esteban Cabral Ortiz y Rosana Jimena Flores ${ }^{2}$
}

\begin{abstract}
Resumen
En los últimos años la perspectiva de interrogar el pasado descubre múltiples pliegues y mediaciones. $\mathrm{Y}$ en ellos se reconocen procesos de re significaciones constantes en los que se articulan distintas formas conocimientos.

En este sentido la propuesta que se presenta surge de la elección de estudiar un lugar histórico en clave arqueológica. Preguntándonos acerca las formas en las que se habitó ese espacio, los lugares que sus habitantes fueron transitando y construyendo lo que hoy conocemos como El monumento Nacional Fuerte de Cobos.

Para su abordaje y comprensión se realizará un relevamiento historiográfico y arqueológico evaluando la documentación histórica en relación a las evidencias materiales surgidas de las excavaciones realizadas en el predio.
\end{abstract}

Palabras clave: Arqueología, Historia, Fuerte de Cobos

\begin{abstract}
In recent years the perspective of interrogating the past from the present discovers multiple folds and mediations. In them, constant resignifications processes are recognized, in which different forms of knowledge are articulated.

In this sense, this proposal comes from the choice to study a place in archaeological key. Wondering about the ways in which the space was inhabited, the places that its inhabitants were transiting and building what today we know as the National Monument of Cobos Fort.

For its approach and understanding, a historiographical and archaeological survey is carried out, evaluating the historical documentation in relation to the evidences arised in the excavations conducted on the property.
\end{abstract}

Keywords: Archeology, History, Cobos Fort

\section{Localización geográfica y contextualización histórica del Fuerte de Cobos}

El Fuerte de Cobos se encuentra emplazado en el extremo oriental del Valle Sianca en la Provincia de Salta en el área comprendida entre la franja de Yungas y parte del umbral del Chaco que es considerada en perspectiva histórica como la frontera oriental del Tucumán (Reboratti, 1990). Los

${ }^{1}$ ICSHO, Facultad de Humanidades Universidad Nacional de Salta. jorgesetbancabral@gmail.com

${ }^{2}$ IEIHis, Facultad de Humanidades Universidad Nacional de Salta. flo.rosana@gmail.com 
ríos que recorren el territorio y los diversos manantiales garantizaron, ya desde el momento de los primeros asentamientos, el riego de parcelas cultivadas de maíz y trigo. La actividad ganadera incluyó ganado vacuno, caballar y la invernada de mula.

Estos valles tenían una buena ubicación en la circulación regional al estar conectados con el camino real que se dirigía al Perú y que bordeaba el Rio Siancas viniendo de Esteco y orientado a Jujuy (Cornejo, 1970, Vitar 2001). Aun cuando a fines del siglo XVII la ruta principal comenzó a pasar por Salta directamente la frontera oriental no perdió sus conexiones con las ciudades. La complementación productiva, los circuitos de intercambio y la presencia de pobladores contribuyeron a un flujo constante de personas y bienes entre las ciudades del Tucumán y la frontera oriental. Entre estos circuitos, el Valle de Siancas permitió el tránsito entre la Gobernación del Tucumán y Charcas actuando a su vez como frontera a través de los asiduos contactos que asumieron tanto la modalidad de cruza y mezcla, como de conflicto (Palomeque, 2009)

La historiografía regional insiste en señalar la importancia que tuvo "la guerra fronteriza contra los indios" en el Tucumán colonial. En efecto, hasta la década de 1770 y con diferentes resultados, desde las ciudades de la gobernación del Tucumán se organizaron acciones punitivas que con el nombre de Entradas Generales que tenían como finalidad "pacificar", "reducir", "sujetar" y "evangelizar" a los indios infieles del Chaco. El peso de estas "entradas" recaía sobre pequeños propietarios rurales, arrenderos o peones reclutados en las ciudades de la gobernación del Tucumán. Los vecinos de las ciudades que participaban de las entradas obtenían "piezas de indios" e incrementaban y "creaban" nutridas fojas de servicios a la Corona que les permitirían ser recompensados con tardías mercedes de tierras. El resultado de estas acciones punitivas conocidas como "entradas" fue el establecimiento de reducciones, muchas de ellas de efímera existencia y de fuertes precarios que servían de abastecimiento.

La instalación de los fuertes e incluso la organización de un cuerpo de soldados partidarios suministrados por las ciudades de la gobernación para defender los fuertes y participar en las entradas, no lograron evitar las incursiones de los pueblos chaqueños sobre la ciudad de Salta. Esta frontera constituyo un área especialmente dinámica que dese fines del XVI fue transitada por una variedad de grupos en interacción social: Indígenas de orígenes diversos en circunstancias cambiantes, y por colonizadores españoles y mestizos que ocuparon el espacio con una serie instalaciones dispersas, las cuales garantizaron la presencia de productores agrícolas y ganaderos, soldados, presidiarios y eclesiásticos seculares y regulares. Los avances de algunas poblaciones chaqueñas sobre este territorio en determinados momentos de la segunda mitad del siglo XVII y la primera parte del XVIII le fueron imprimiendo una condición de inestabilidad de los establecimientos y la vida de sus habitantes (Mata, 2005).

En el marco de estos procesos de conquistas y colonización en el valle de Siancas se produjo la creación del Fuerte de Cobos. Probablemente que su fundación fuera realizada por el teniente gobernador de Salta, Diego Gómez en el año 1690 el que le asignó el nombre de Fuerte de Santa Ana (Zorreguieta, Ibarguren, 2008). En este proceso, Cobos formaba parte de una línea de fortificaciones, que en muchos casos consistían en pequeñas construcciones de tapiales que funcionaban como guarnición militar y que fueron creados en la necesidad de contener el avance de los grupos indígenas chaqueños. Entre estos pueden destacarse los fuertes de Balbuena y San José los cuales fueron destruidos en 1775 por una invasión indígena.

Muchos historiadores tradicionales han utilizados estos datos para consolidar una imagen del fuerte como baluarte en contra de la ofensiva indígena y como enclave estratégico en las guerras de independencia (Geres, 2012). Sin embargo, Cobos pareciera haber tenido también un rol destacado en el camino de postas reales dada su mención y descripción en 1772 en los escritos de Alonso Carrió de la Vandera. Para ese entonces la propiedad y vivienda familiar eran de Rosalía Martínez y de su esposo Francisco Xavier de Olivares encargados de mantener en funcionamiento ese enclave del camino real el cual servía como última parada en el acceso a la ciudad de Salta. 
Este carácter de posta, el cual brinda un aspecto distinto al de fuerte, se halla descrito en las crónicas de viajes de Edmundo Temple, un cronista que transita por el lugar en 1826 en sus viajes por las postas comprendidas entre los caminos de Salta, Jujuy y Tucumán. En este escrito se describe el acceso por el poblado que rodeaba al fuerte el cual se encontraba en malas condiciones, destacando la falta de abastecimiento para los viajantes (Temple, 1826).

Sin lugar a dudas las transformaciones económicas y ocupación espacial en el siglo XIX le fue otorgando una impronta que muy poco aborda desde los estudios históricos. Los cuales se ocuparon en resaltar, que en dos oportunidades el general Manuel Belgrano se hospedó en este edificio. La primera fue el 26 de agosto de 1812, cuando realizó el éxodo de Jujuy y se retiraba hacia el sur. La última oportunidad fue el 14 y 15 de febrero de 1813, antes de dirigirse hacia Salta, donde lo esperaba la Batalla del 20 de Febrero. Durante la Guerra Gaucha el fuerte fue utilizado como cuartel por parte del general Martín Miguel de Güemes. El 15 de junio de 1816 en este lugar, el director supremo de las Provincias Unidas del Río de la Plata, Juan Martín de Pueyrredón le encomienda a Güemes mantener alejado al ejército Realista, para que en Tucumán se reunieran los congresales y se deliberara la ansiada Independencia Nacional.

Estos acontecimientos condicionaron su valor arquitectónico e histórico por el que fue declarado Monumento Histórico Nacional el 14 de Junio de 1941.

\section{Excavaciones en el predio del Fuerte de Cobos}

Las perspectivas arqueológicas y sus metodologías de análisis nos permiten acceder a otra forma en la que puede construirse el tiempo histórico. En la que se conjugan los análisis documentales y el de los objetos que las personas utilizaron, ofreciendo un camino en la reflexión acerca de cómo se ocuparon y construyeron los espacios. El análisis de las fuentes materiales, muchas veces nos permiten descubrir lugares vigentes en la memoria y presentes desde la materialidad en los que se llevaron a cabo las actividades cotidianas de sus pobladores. De esta manera, puede complejizarse la perspectiva de la historiografía local, la cual resalta y ata ciertos lugares a hechos nacionales enaltecidas por las gestas militares para empezar a acceder de manera más significativa a los espacios construidos desde la practica social.

Durante el último año, un grupo de estudiantes e investigadores de la UNSa decidimos estudiar un lugar histórico en clave arqueológica. Preguntándonos sobre las formas en las que se habitó ese espacio, tratando de comprender esos los lugares que sus habitantes fueron transitando y con ello construyendo lo que hoy conocemos como El monumento Nacional Fuerte de Cobos. En la actualidad, éste se corresponde a un edificio de dos pisos, de paredes de adobe dispuesta en un predio delimitado por un muro perimetral que cierra un espacio rectangular en cuyos vértices se observan pequeñas estructuras de diamantes que insinúan la forma típica de un fuerte hispano. Esto supuso un rasgo significativo dado que en las descripciones realizadas por los viajantes (Concolorcorvo y Edmundo Temple) nunca se menciona la presencia de estos muros sino que se hace alusión a la estructura que hoy se conserva insinuando la posibilidad de que existieran otros espacios que sirvieran para el funcionamiento del lugar como posta de camino.

Desde aquí surgió la necesidad de indagar como fue que se ocupó ese espacio declarado Monumento Histórico Nacional, preguntándonos si en las sucesivas remodelaciones se dejaron de lado sectores habitacionales que pudieron funcionar en los siglos XVIII y XIX y que fueran ignorados en las declaratorias nacionales del siglo XX. En este marco es que se decidió alternar entre dos vías metodológicas el análisis de documentos en discusión con las tareas de campo.

Por ello es que se introdujo el análisis el registro fotográfico proveniente del fondo documental de Arquitectura de la Nación a través del cual pudimos destacar lo sucedido durante las diferentes remodelaciones del edificio principal. En vista de poder acceder a una comprensión desde lo material 
fue que pudimos acceder a diferentes documentaciones fotográficas y de planimetría que nos permitió registrar las sucesivas remodelaciones que afectaron la estructura desde antes de su declaración. Aquí el énfasis estuvo dado en poder tratar de detectar posibles espacios que hayan sido olvidados en las sucesivas restauraciones y que puedan haber sido utilizado en momentos de la ocupación del Fuerte. Luego decidimos realizar un relevamiento arqueológico, se realizaron prospecciones que permitieran dar cuenta de estructuras habitacionales y que hayan quedado enterradas luego de las constantes intervenciones. El relevamiento arqueológico nos permitió detectar dentro del predio sectores con material en superficie, alejados de la estructura principal que formaban conjuntos de fragmentos de lozas inglesas propias del siglo XIX, de ladrillos y vidrios asociados a eventos de remoción recientes. Sumado a esto se registró rocas alineadas que sugerían la presencia de algunas estructuras arqueológicas muchas de las cuales se hallaban también cercanas a la casa principal.

Esto nos permitió pensar en las posibilidades de ocupación durante el siglo XIX la cual pudiera también registrarse en algunos documentos fotográficos de la época. Por ello decidimos realizar una primera excavación en el sector Suroeste, al costado de la casa principal, siguiendo una línea rocas (Figura 1).

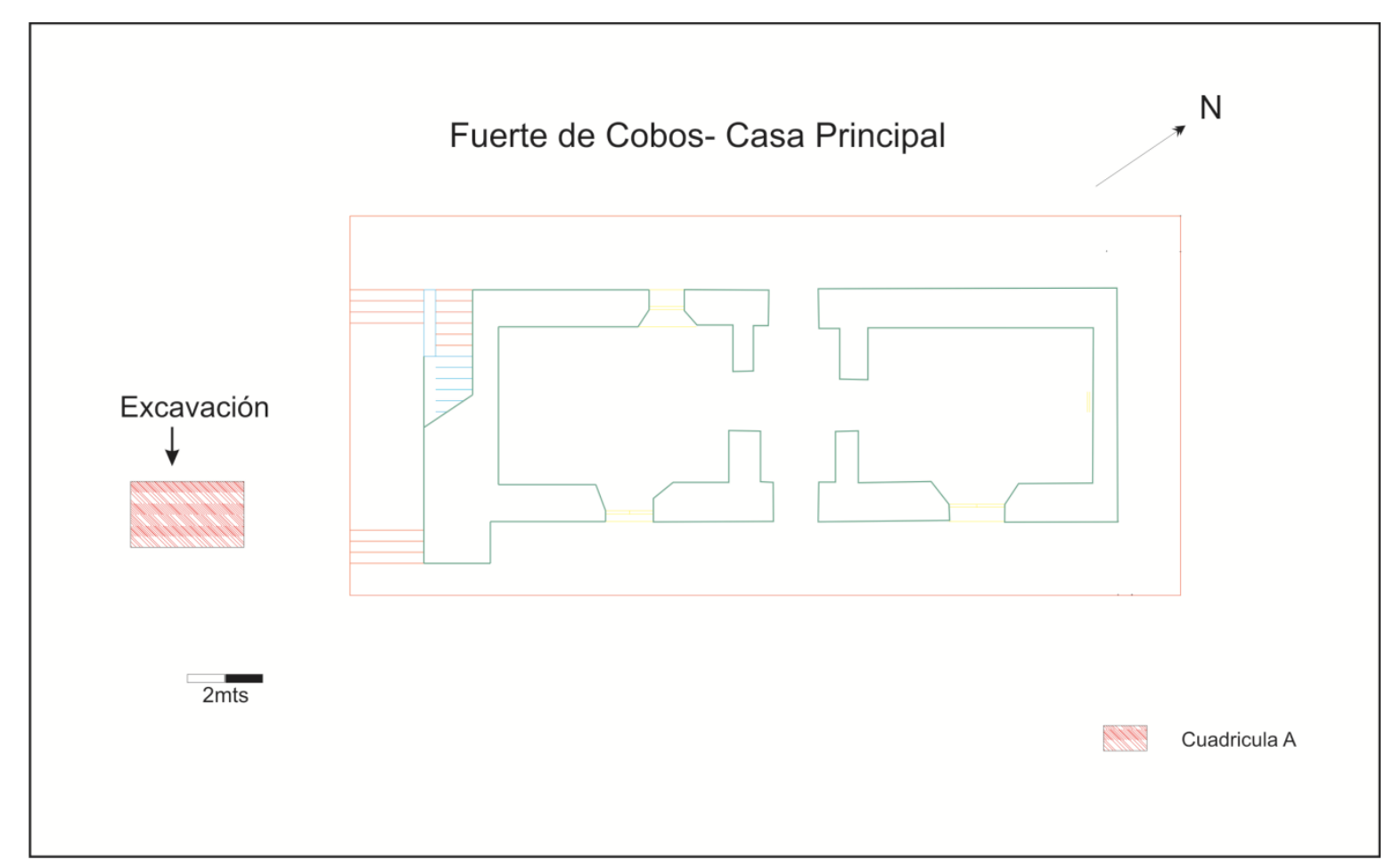

Figura 1: Plano de la casa principal Fuerte de Cobos en el que se muestra el sector excavado

La cuadrícula, de $3 \mathrm{mts}$ X $2.80 \mathrm{mts}$ fue excavada a través de la técnica décapage siguiendo la secuencia estratigráfica natural. A través de la misma se pudo identificar la presencia una antigua estructura habitacional la cual pudo estar funcionando entre finales del siglo XIX y principios del XX (Figura2). Esta se define por un muro de piedra unida con barro batido, que se prolonga en un cimiento por debajo del posible piso de ocupación. Probablemente la mitad superior del muro que conformó esta habitación se encontraba conformado por hileras de adobe unidas con barro batido. De acuerdo a las lecturas estratigráficas se pudo identificar 3 unidades de procedencia, las cuales contenían diferentes tipos de materiales de construcción, fragmento de adobe, piedra de derrumbe, etc. Posiblemente éstas, estén haciendo referencia a los diferentes episodios de derrumbe de la men- 
cionada habitación. Contenidas en las tres unidades estratigráficas se pudo recuperar un total de 200 fragmentos óseos muchos de los cuales remiten a partes de animales vacunos. Como así también un fragmento de caparazón de quirquincho carbonizado.

Respecto a los conjuntos cerámicos analizados, todos ellos corresponden a cerámica de terracota sin decoración mientras que en un solo caso pudimos detectar cerámica de color verde vidriada. Estas cerámicas se asemejan a aquellas de manufactura jesuita y posiblemente su uso se encuentre extendido entre fines del siglo XVII y XIX.
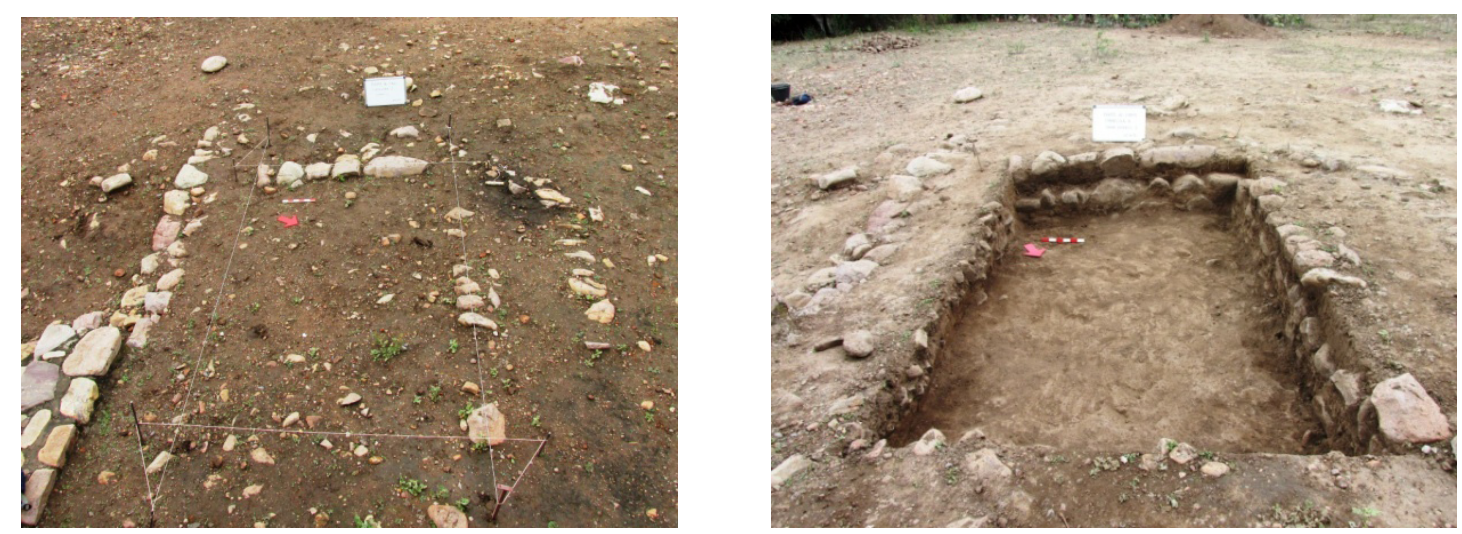

Figura 2. Cuadricula de excavación en la que se observa la línea de muro de la estructura asociada a la casa principal en el Fuerte de Cobos

Los datos obtenidos desde nuestra primera excavación pudieron ser cotejados a partir del análisis de los documentos fotográficos. En el archivo de la Provincia de Salta pudimos acceder a una fotografía de 1925 en la que se observan un grupo de personas dispuestas en la planta alta y baja del edificio principal y dos carruajes de época. En ella puede observarse a la izquierda una antigua estructura de paredes de adobes la cual pareciera estar en uso. Por otro lado, en el registro fotográfico del archivo de Arquitectura de la Nación, pudimos registrar una fotografía de 1942 en la que se observa la desaparición de esa estructura, la cual pareciera estar sepultada por el derrumbe de la escalera de acceso a la segunda planta.

De acuerdo al registro fotográfico, es posible de suponer que la estructura habitacional excavada haya estado en funcionamiento entre finales del siglo XIX y comienzos del XX. Probablemente que este espacio haya funcionado como un sector vinculado a la casa principal, sin embargo, el escaso material recuperado al interior de la misma no permite especificar su uso. De las lecturas estratigráficas se pudo identificar 3 capas de las que provienen diferentes tipos de materiales de construcción, fragmento de adobe, etc. haciendo referencia a los diferentes episodios de derrumbe de la mencionada habitación. 

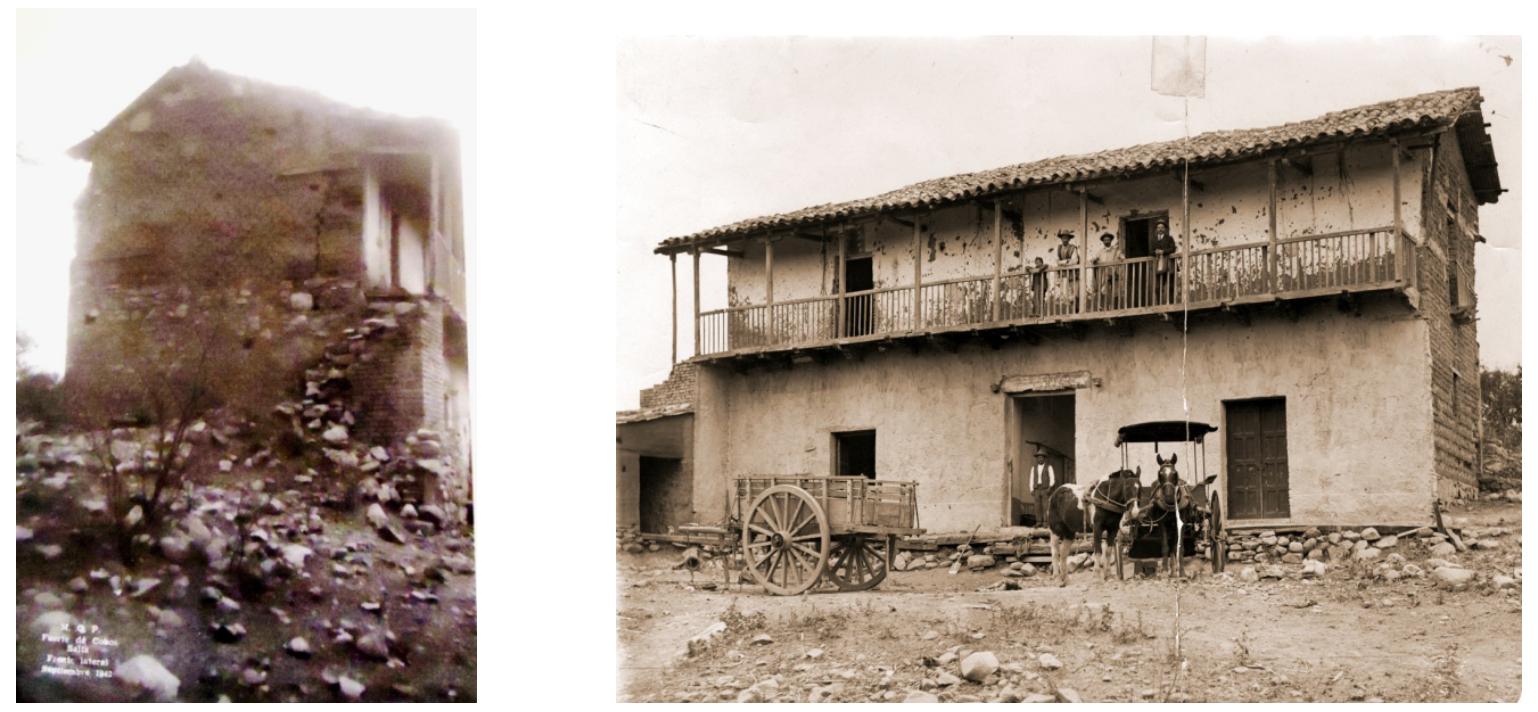

Figura 3. Derecha foto de 1925 en la que puede observarse parte de la estructura excavada en funcionamiento. Izquierda, foto de 1942 en la que se observa el episodio de derrumbe que sepultó la estructura excavada

\section{Consideraciones finales}

En esta propuesta de estudio se buscó relacionar entre dos metodologías de análisis, por un lado, el estudio documental y por otro el de las materialidades. En este sentido, ambos registros permitieron abordar un mismo espacio desde diferentes disciplinas científicas.

Desde la perspectiva histórica los análisis centrados en los estudios de las fronteras como espacios porosos de interacción social y cultural permiten una nueva mirada historiográfica a través de estudios regionales y locales que abren nuevos problemas y perspectivas. Este primer acercamiento al Fuerte de Cobos nos permite suponer diversas modalidades de poblamiento en dicho espacio enmarcadas en las estructuras socioeconómicas desplegadas para cada siglo de ocupación. Dentro de estos últimos, se debe enfatizar el rol jugado por las diferentes coyunturas de migraciones, que van desde los asentamientos indígenas y las economías agrarias coloniales hasta las nuevas producciones exportadoras del S.XIX. Es precisamente este siglo el que nos presenta un desafío para seguir indagando en ambos registros: histórico y arqueológico.

Por otro lado, el análisis de las materialidades nos permitió dar cuenta de espacios habitacionales muchas veces olvidados desde la historiografía tradicional. Aquí cobra relevancia el registro de una estructura anexada a la casa principal a la cual no pudimos atribuir una funcionalidad específica pero que pareciera haber estado en uso entre finales del siglo XIX y comienzo del siglo XX. Esto pone en relieve las perspectivas basales que llevaron a la declaratoria de Monumento Histórico Nacional de lo que hoy se conoce como El Fuerte de Cobos. Sin lugar a dudas que esta declaratoria en la primera mitad del siglo XX, estuvo condicionada por sus valores arquitectónicos e históricos en la necesidad de recuperar espacios olvidados en el tiempo.

Según los resultados de la excavación, en esta tarea de rescate y puesta en valor del pasado no se contemplaron espacios que pudieron haber servido en las labores cotidianas de aquellos pobladores. Aquí resulta llamativo la construcción del muro perimetral por parte de los organismos Nacionales, dado que esta estructura nunca fue mencionada por los relatos de viajeros. Según lo indagado en los archivos de Arquitectura de la Nación este modelo de muro defensivo fue tomado de los fuertes jujeños que quedaron en pie. 
A partir de esta primera propuesta de estudio del Fuerte de Cobos es que nos surgen nuevas líneas de investigación, como también indagar sobre las configuraciones del pasado histórico y la necesidad de patrimonializar ciertos espacios de la historia local en un marco de emergencia de nuevas identidades. En este sentido, el hallazgo de una casa de una estructura habitacional ocupada desde finales del siglo XIX y su olvido en las sucesivas intervenciones de restauración pone en relieve un sentido del pasado por parte de los organismos nacionales, el cual pareciera estar basado en la necesidad de resaltar las gestas militares vinculadas a las guerras de independencia.

\section{Agradecimientos}

Al personal de Arquitectura de la Nación por permitirnos acceder a los archivos fotográficos. A los alumnos de la cátedra Métodos y Técnicas de la investigación de la Facultad de Humanidades y a la profesora María Clara Rivolta por colaborar en las tareas de excavación. Al personal del Museo de Campo Santo por su acompañamiento en la logística en las tareas a campo.

\section{Referencias}

CONCOLORCORVO. (1997). Lazarillo de los ciegos caminantes 1776. Buenos Aires: Ed Emece.

CORNEJO, A. (1945). Contribución a la historia de la propiedad inmobiliaria de Salta en la época virreynal, Buenos Aires: Instituto de Investigaciones Históricas San Felipe y Santiago

MATA DE LÓPEZ, S. (2005). Las fronteras coloniales como espacios de interacción social. Salta del Tucumán (Argentina), entre la Colonia y la Independencia. Dimensión Antropológica, 33, 69-90.

GEREZ, O. (2012). Los desaires de la memoria, Cobos un fuerte histórico y una historia a medias tintas. Revista Claves.

PALOMEQUE, S. (2009). Los caminos del sur de Charcas y de la Gobernación el Tucumán durante la expansión inca y la invasión española (siglos XV-XVII).

REBORATTI, C. (1997). De hombres y tierras. Una historia ambiental del noroeste argentino, Salta, Proyecto Desarrollo Agroforestal en Comunidades Rurales del Noroeste Argentino. En Carlos Reboratti (Comp.) La diversidad ambiental del Noroeste. (pp. 11-25) Salta: GTZ

TEMPLE E, (1826). Córdoba, Tucumán, Salta y Jujuy en 1826. Tucumán: Universidad Nacional de Tucumán, Argentina: Ediciones de Rectorado.

VITAR, B. (1997). Guerra y misiones en la frontera chaqueña del Tucumán (1700-1767). Madrid: CSIC.

ZORREGUIETA, M. y IBARGUREN, A. (2008). Apuntes Históricos de la Provincia de Salta en la época Del Coloniaje. Colección Artes y ciencias. Serie Historia. (pp. 23-27). Editorial EUCASA / B.T.U.

Recibido: 11/06/2017

Aceptado: 03/09/2017 